load is associated, externalization or internalization. That is, in order to determine whether pupillary dilation is an attentional or motivational reaction, it is necessary to specify whether the task requirement calls for externalization or internalization and to determine if pupillary orienting reflex to stimulus novelity is inductively related to the direction of the reflex in terms of task requirement.

\section{REFERENCES}

Bradshaw, J. L. Load and pupillary changes in continuous processing tasks. British Journal of Psychology, 1968, 59, 265-271.

Coleman, F., \& Paivio, A. Pupillary dilation and mediation processes during paired-associate learning. Canadian Journal of Psy chology, 1970, 24, 261-270.

Gardner, R. M., Mo, S. S., \& Borrego, R. Inhibition of pupillary orienting reflex by novelity in conjunction with recognition memory. Bulletin of Psychonomic Society, 1974, 3, 237-238. Kahneman, D., \& Beatty, J. Pupil diameter and load on memory. Science, 1966, 154, 1583-1585.

Kahneman, D., Beatty, J., \& Pollack, I. Perceptual deficit during a mental task. Science, 1967, 157, 218-219.

Pavlov, I. P. Conditioned reflex. Oxford University Press, 1927 (Dover, 1960).

Sokolov, Ye. N. Perception and the conditioned reflex. New York: Pergamon, 1963.

Thorndike, E. L., \& Lorge, I. The teacher's word book of 30,000 words. New York: Teachers College Press, 1944.

(R eceived for publication August 5, 1974.)

\title{
A component analysis of natural language mediators obtained in paired-associate learning*
}

\author{
JERRY M. OWENS $\dagger$, PAMELA R. WERDER, and PHILIP H. MARSHALL \\ Texas Tech University, Lubbock, Texas 79409
}

\begin{abstract}
The structural relationships between paired-associate components and natural language mediators (NLMs) were analyzed by using forward and backward recall paradigms. Ss were required to give the NLM and the appropriate to-be-recalled component for pairs learned by natural language mediation. An insignificant difference was found in the ability of "stimulus" and "response" components to elicit NLMs, but a significant difference was obtained in the ability to decode the NLM into the correct response, with the forward recall procedure resulting in more efficient response recall. The results were discussed in terms of differential elaboration of stimulus and response components and in terms of Adams's (Adams \& Bray, 1970) theory of paired-associate learning.
\end{abstract}

In Adams's closed-loop theory of paired-associate (PA) verbal learning (Adams \& Bray, 1970), a natural language mediator (NLM) is considered to be any idiosyncratic verbal device which $\mathrm{S}$ uses to mediate the learning of a stimulus-response pair. Natural language mediators used during learning are detected by verbal reports. The use of NLMs has been found to increase recall in short- and long-term retention (Adams, Marshall, \& Bray, 1971a, b; Groninger, 1966; Kiess, 1968; Montague, Adams, \& Kiess, 1966) and to reduce interlist interference (A rams et al, 1971a; Adams \& Montague, 1967).

* This research was supported in part by funds from the Graduate School, Texas Tech University. The paper is sponsored by C. G. Halcomb, who takes full editorial responsibility. Requests for reprints should be sent to Philip $H$. Marshall, Department of Psychology, Texas Tech University, Lubbock, Texas 79409

+Now at the Naval Aerospace Medical Research Laboratory, Pensacola, Florida.
By Adams's (Adams \& Bray, 1970) definition, a NLM embodies both the stimulus and response terms and is considered as a response itself. The implication is that a NLM, once established, can be elicited by either the stimulus or response term. Adams allowed, however, that NLMs may have a favored direction (i.e., forward) which could suggest the possibility of greater ease of decoding the NLM for the correct response when forward rather than backward recall is required.

The purpose of this investigation was to analyze structural relationships between PA components and NLMs. To determine whether both members of the PA item are equally capaple of eliciting the common NLM, the procedures of forward and backward recall were used. In addition to response recall, Ss were required to recall the actual NLMs to determine NLM availability. They were then to recall the responses, presumably requiring decoding of the NLM, which resulted in 
information concerning NLM decodability. An analysis was then made of the NLMs themselves, and factors contributing to the development of a "favored direction" were investigated.

\section{METHOD}

\section{Experimental Design}

Eighty-six students in introductory psychology classes participated in the experiment for course credit. Ss were randomly assigned to two recall groups, A-B and B-A, with an approximately equal number of men and women in each group. Both learned a list of eight CVC pairs in the forward direction by the correction method and were free to learn the items by mediation or by rote. Following acquisition, an interpolated arithmetic task was performed by all Ss for $10 \mathrm{~min}$, and a recall test was given immediately after the arithmetic task. On this final recall test, Group A-B was asked to recall NLMs and B terms when the A terms were presented. Group B-A was asked to recall NLMs and A terms when given the B terms for the pairs. There were no treatment differences in the groups prior to the retention task.

\section{Apparatus and Materials}

A Kodak Carousel projector, controlled by a Varichron V3 timer presented the slides containing the CVC pairs. A remote switch allowed the projector to be manually advanced by $\mathrm{E}$ and the timer to be simultaneously reset. The CVC pairs, selected from the norms of Montague and Kiess (1968), had a mean associability value of 48 , with a range of 46 to 51 . Each CVC was used only once in the entire list. A t test on the associative values of CVCs used for A and B terms (mean $=41.00$ for $\mathrm{A}$ terms, 41.25 for B terms) revealed no significant differences.

\section{Acquisition}

Two trials by the correction method were given with the test phase requiring A-B recall. ${ }^{1}$ A correction trial requires a series of study-test sequences until one correct response is given for each pair. In the study portion of the first sequence, all pairs were presented once for study and all A terms were presented once for test. All pairs having correct responses were removed from the second study-test sequence. The procedure continued until one correct response had been made for each pair, ending that particular trial. Two random orders of pair presentation for the first study phase of each correction trial were predetermined. Half of the Ss in each recall group received one order, while the other half received the second order for the two acquisition trials. There was a maximum of $10 \mathrm{sec}$ allowed for each pair in the study and test phases, with the slide being manually advanced during the test phase immediately after any response was given. Ss were required to spell aloud each CVC pair during the study phase and also to report aloud NLMs as soon as they occurred. Between study and test phases, $\mathrm{S}$ read aloud single digits from two slides presented for $10 \mathrm{sec}$ each.

Recorded instructions were the same for all Ss, explained the procedures of PA learning by the correction method, and discussed rote and mediated learning without emphasizing either procedure. Natural language mediators were recorded for each mediated item in the study sequence. Acquisition practice on two nonexperimental pairs was given in order to check S's understanding of the procedures. In the initial instructions, there was no mention of the interpolated arithmetic task or of the retention test to follow.

\section{Retention Test}

After the 10-min arithmetic task, recorded instructions for half of the Ss (designated Group A-B) informed S that he would receive one test trial on each of the eight CVC pairs learned earlier. As in the tests during acquisition, Ss of Group A-B were shown the $A$ term and were asked to give the $B$ term. If any NLM was reported during original learning (defining that particular item as a mediated item), $\mathrm{S}$ received two test slides for that pair, each showing the A term. The first presentation was for the recall of the NLM and the second for the recall of the B term. Ten seconds were allowed for recall of the NLM and an additional $10 \mathrm{sec}$ for recall of the $\mathrm{B}$ term, but the slides were manually advanced as soon as $S$ made his reports. Both NLMs and responses were recorded by $\mathrm{E}$. Instructions for Group B-A were exactly the same as those for Group A-B, with the exception that A-term recall was required.

\section{RESULTS AND DISCUSSION}

The data in Table 1 indicate superiority of forward over backward recall and superior recall for mediated items. Moreover, the dependency on correct NLM recall for correct response recall of mediated items is clearly indicated, and this has been reported elsewhere (Adams \& Bray, 1970; Adams et al, 1971a, b). This dependence on NLM recall for mediated items also suggests that for mediated items differences between A-B and B-A recall may be influenced by factors other than superior response learning per se and point to the need for the NLM analyses presented below. Table 1 also indicates that Ss who were to be given B-A recall were more likely to use a mediation strategy during acquisition. However, since acquisition instructions and procedures were identical for the two groups, this was treated as a chance occurrence and unrelated to the mediational strategies of the two groups.

\section{NLM Analyses}

The central analyses concentrated on the availability and decodability of NLMs under each condition. The proportion of NLMs recalled correctly, for those Ss with at least one mediated item, was included in the analysis. ${ }^{2}$ Group A-B contributed 35 Ss, while Group B-A contributed 38 Ss. The difference between the means $\overline{\mathrm{X}}_{\mathrm{A}-\mathrm{B}}=.82, \overline{\mathrm{X}}_{\mathrm{B}-\mathrm{A}}=.73$ ) was found to be nonsignificant $[\mathrm{t}(71)=1.65]$, indicating that the correct recall of NLMs was not significantly affected by recall order.

Natural language mediators may also differentially

Table 1

Proportion of Responses Recalled Correctly as a Function of Item Classification and Recall Order

\begin{tabular}{|c|c|c|c|c|}
\hline \multirow{3}{*}{$\frac{\text { Item Classification }}{\text { Rote in Acquisition }}$} & \multicolumn{4}{|c|}{ Recall Order } \\
\hline & \multicolumn{2}{|c|}{$A-B$} & \multicolumn{2}{|c|}{ B-A } \\
\hline & .83 & $(175)^{*}$ & .65 & (124) \\
\hline $\begin{array}{l}\text { Mediated in Acquisition } \\
\text { (NLM Correct on Recall) }\end{array}$ & .95 & (138) & .82 & (164) \\
\hline $\begin{array}{l}\text { Mediated in Acquisition } \\
\text { (NLM Incorrect on Recall) }\end{array}$ & .26 & $(31)$ & .18 & $(56)$ \\
\hline
\end{tabular}

*Numbers in parentheses give number of observations for denominator. 
influence A-B and B-A response recall by their decodability in each condition. Decoding a NLM refers to the translation of the NLM into its appropriate original components. Using only correctly recalled NLMs, the proportion that were correctly decoded by each $\mathrm{S}$ in each group (34 Ss in A-B and 37 Ss in B-A) was entered in a $t$ test comparison. The results indicated that correctly recalled NLMs were more likely to be correctly decoded in the A-B condition than in the B-A condition $[t(69)=2.74, p<.01]$, with the mean proportion correct for Group A-B being .94 and for Group B-A being .81 .

The results of the availability analysis indicated that the availability of NLMs was not significantly different under A-B and B-A conditions. This finding supports Adams's notion that NLMs may be elicited by either the stimulus or response member of a PA item, since the NLM is, by definition, composed of elements of both. However, Adams also indicated that NLMs may have a favored direction, and the results of the decodability analysis supported that argument as well. In conjunction with the decodability hypothesis, an inspection of the nature of the recall errors in each recall condition, given correct NLM recall, revealed only two errors of commission in the A-B condition, while 30 errors of commission were found in the B-A condition. An error of commission was most frequently characterized as being a response very close to the original CVC but, typically, either two or three correct letters of the CVC were rearranged or one incorrect letter was substituted for a correct letter.

One possible explanation for the more accurate decodability of NLMs in A-B recall has to do with A-term and B-term elaboration. The demands of the acquisition procedure required all Ss to give a response upon presentation of a stimulus. Given such a requirement, Ss may adopt a NLM-producing strategy that yields a greater modification or elaboration of the $\mathrm{A}$ term while keeping the B term relatively intact. Elaboration refers to modification or alteration of the CVC which leads to increased distortion of the nominal CVC. Bower (1967) has made a similar statement: "Poorly integrated stimuli lead to poor backward recall because the mnemonics encode only a few aspects of the full stimulus term [p. 192]." In order to test this explanation and to "dissect" the NLMs resulting from PA learning, a procedure developed by Prytulak (1971) was adapted for use in the present study.

Prytulak's model was based on the number and type of transformations used to reconstruct a single CVC from NLMs written by the S. To apply Prytulak's classification to the present PA data, the A and B terms for a given pair were treated separately. Single-word NLMs based on both A and B terms were eliminated. Since $S$ reported his NLMs verbally, any uncertainty on the spelling was resolved by the judgment of two Es. A $96.4 \%$ agreement was initially realized, and discussion between the two Es resolved the remainder.

If the assumption that transformations associated with stimulus terms are more highly elaborated than those used with response terms is true, then differences should be noted between the number of operations to produce stimulus and response transformations. The rationale, then, was that a larger number of operations should be associated with greater elaboration. This in turn would lead to more difficulty in decoding. This follows from Prytulak's finding that, in general, the more operations used for a particular NLM, the less accurate was decodability (1971, Fig. 1). A two-tailed $t$ test for correlated samples on the number of operations involved in forming $\mathrm{A}$ - and B-term transformations for all acceptable mediated items showed a significant difference $[\mathrm{t}(244)=4.06$, $p<.001]$. The mean number of operations used for A-term transformations was 1.61 , while the mean number used for B-term transformations was 1.26.

The results of the preceding analyses provide support for the argument that NLMs in A-B recall are easier to decode due to the less elaborated, or modified, status of that portion of the NLM associated with the response CVC. Consequently, the mechanics of Adams's "favored direction" which NLMs may take seem to be embodied in the predominant NLM-producing strategies which Ss either bring with them to the experimental setting or which are induced by the task demands.

\section{REFERENCES}

Adams, J. A., \& Bray, N. W. Closed-loop theory of paired-associate verbal learning. Psychological Review, 1970 $77,385-405$.

Adams, J. A., Marshall, P. H., \& Bray, N. W. Interference and retrieval. Journal of Verbal Learning \& Verbal Behavior, $1971 a, 10,548-555$.

Adams, J. A., Marshall, P. H., \& Bray, N. W. Closed-loop theory and long-term retention. Journal of Experimental Psychology, $1971 \mathrm{~b}, 90,242-250$.

Adams, J. A., \& Montague, W. E. Retroactive inhibition and natural language mediation. Journal of Verbal Learning \& Verbal Behavior, 1967, 6, 528-535.

Bower, G. H. Verbal learning. In H. Helson and W. Bevan (Eds.), Contemporary approaches to psychology. Princeton: Van Nostrand, 1967.

Groninger, L. D. Natural language mediation and covert rehearsal in short-term memory. Psychonomic Science, 1966, 5, in short-136.

Kiess, H. O. Effects of natural language mediators on short-term memory. Journal of Experimental Psychology, 1968, 77, 7-13.

Montague, W. E., Adams, J. A., \& Kiess, H. O. Forgetting and natural language mediation. Journal of Experimental Psychology, 1966, 72, 829-833.

Montague, W. E., \& Kiess, H. O. The associability of CVC pairs. Journal of Experimental Psychology, 1968, 78(2, Pt. 2).

Prytulak, L. S. Natural language mediation. Cognitive Psychology, 1971, 2, 1-56.

\section{NOTES}

1. The correction procedure was used to provide the same criterion of learning for all pairs.

2. Past research (Adams et al, 1971a. b) has found correlations greater than $x=.95$ when two judges rated correctness of NLM recall up to several months after completion of the experiment. Therefore, in this study $\mathbf{E}$ was allowed to make that decision during the actual retention test. 